\title{
Islas de fertilidad: una revisión sistemática de su estructura y operación
}

\author{
Fertility islands: a systematic revision of Structure and operation
}

María Cristina Moncayo-Riascos ${ }^{1 *}$, Arturo Gálvez-Cerón ${ }^{2}$

\section{RESUMEN}

En ecosistemas áridos y semiáridos, las islas de fertilidad o de recursos están conformadas por un mosaico de vegetación arbustiva dispersa, rodeada por una matriz de suelo deteriorada desde el punto de vista productivo. Los resultados de diferentes estudios sugieren que los arbustos están directamente relacionados con la dinámica de formación y los patrones de acumulación de nutrientes, controlando funciones a nivel micro y macroecológico. El objetivo de este trabajo es ofrecer un acercamiento al concepto de islas de fertilidad por medio del método de revisión sistemática. El resultado incluyó el análisis de 18 estudios con información relevante acerca de las causas biofísicas y socioecológicas de formación de las islas, la estructura característica de los estratos herbáceo y arbustivo, y el rol espacio-temporal que las comunidades de microorganismos, artrópodos y fauna juegan en su formación. Además, se identificaron las funciones de las islas de fertilidad propuestas en la literatura científica y sus diferentes enfoques. Finalmente, se planteó a futuro el estudio de las islas bajo el enfoque de sistemas complejos y el concepto de resiliencia socioecológica.

Palabras clave: paisaje, reserva de nutrientes, sucesión natural, ecosistema semiárido.

\begin{abstract}
In arid and semi-arid ecosystems fertility or resource islands are made up of a mosaic of scattered shrub, surrounded by a deteriorated soil matrix from the production point of view. Results of several studies suggest that the shrubs are directly related with the dynamics of formation and nutrient accumulation patterns, controlling functions at a micro and macro-ecological level. The aim of this document is to provide an approach to the concept of fertility islands through the systematic revision method. The result included an analysis of 18 studies with relevant information on biophysical and socio-ecological formation causes, the characteristic structure of the herbaceous and shrub layers, and the spatio-temporal role of communities of microorganisms, arthropods and fauna. The functions of the islands of fertility proposed in the scientific literature and their different approaches were identified. In conclusion, the study of the islands under the complex system approach and the socio-ecological resilience concept were proposed for the future.
\end{abstract}

Key words: landscape, nutrient reserve, natural succession, semi-arid ecosystem.

\section{Introducción}

Los ecosistemas de zonas áridas y semiáridas se caracterizan por una distribución heterogénea de recursos (Housman et al., 2007; Xu et al., 2012), y una limitada productividad primaria. Esto debido a la alta radiación solar, las altas tasas de evapotranspiración, las restricciones en la disponibilidad del agua y la baja movilidad de nutrientes en el suelo, especialmente de nitrógeno y fósforo (Dossa et al., 2009; Ruiz, et al. 2008).
Las especies como resultado de estas características de los ecosistemas se encuentran en estado latente durante los períodos más secos del año, y se activan con los primeros eventos de lluvia (Huxman et al., 2004), creando un mosaico de vegetación dispersa denominado islas de fertilidad, fértiles, o de recursos (Dossa et al., 2009; Housman et al., 2007).

Las islas de fertilidad son patrones de vegetación rodeados por suelo descubierto, que inducen bajo las plantas la acumulación y el

1 Universidad de Antioquia, Ciudad Universitaria Calle 67 Nº 53-108. Bloque 5, oficina 418 apartado aéreo: 1226. Medellín, Colombia.

2 Universidad de Nariño, Facultad de Ciencias Pecuarias: calle 18 No 52-35 Ciudad Universitaria Torobajo, Pasto-Colombia.

* Autor para correspondencia: maria.moncayo@udea.edu.co

Fecha de Recepción: 25 Julio, 2017.

Fecha de Aceptación: 10 Octubre, 2017. 
enriquecimiento de recursos (Ruiz et al., 2008; Li et al., 2013; Yang et al., 2011a). Dicho patrón configura una complejidad estructural que soporta una alta diversidad y abundancia relativa de plantas (Zaady et al., 2013a; Alhamad y Alrababah, 2013).

En particular, las investigaciones sobre islas de fertilidad se han enfocado en estudiar su estructura y la dinámica ecológica que genera dentro de los ecosistemas áridos o semiáridos.

Con respecto a la estructura, las investigaciones han concentrado su esfuerzo en el análisis de factores físicos y biológicos de su formación (Allington y Valone, 2013; Housman et al., 2007; F.-R. Li, et al., 2012), los patrones de nutrientes del suelo alrededor de los arbustos (Alhamad y Alrababah, 2013; Caballero et al., 2007; Zaady et al., 2013a; Allington y Valone, 2013), la composición y estructura de la vegetación (C. Li et al., 2013; $\mathrm{Xu}$ et al., 2012), la complejidad estructural y su continuidad a través del tiempo (Alhamad y Alrababah, 2013; DeMars et al., 2010; Manning et al., 2006).

Por otra parte, los estudios enfocados en las dinámicas generadas entorno a las islas han estudiado las funciones ecológicas de los pequeños mamíferos (Xu et al., 2012), la biodiversidad en los parches de vegetación, las modificaciones en el microambiente (Alhamad y Alrababah, 2013; Dossa et al., 2009; F.-R. Li et al., 2012), y los procesos hidrológicos de los ecosistemas semiáridos (Zaady et al., 2013b), que influyen en el gradiente de potencial hídrico (Qinmei et al., 2007).

Esto evidencia, que el estudio de las islas de fertilidad se ha realizado desde disciplinas como la biología, la ecología, la hidrología, la edafología y la mineralogía de manera independiente, y desde perspectivas y unidades de análisis desagregadas. En este contexto, el trabajo busca ofrecer una revisión en conjunto de la estructura y funcionamiento de las islas de fertilidad, por medio del método de revisión sistemática de literatura científica.

Los resultados incluyen el análisis crítico de 18 investigaciones, desarrolladas en entornos áridos y semiáridos de nueve países, consolidando las diferentes acepciones, escalas y funciones de las islas de fertilidad. Finalmente, se plantea la necesidad de valorar las interrelaciones y posibles estrategias para su gestión desde el enfoque de sistemas complejos y el concepto de resiliencia socioecológica.

\section{Materiales y Métodos}

La revisión sistemática incluyó estrategias para la recolección, valoración, selección crítica y síntesis de artículos tomando como referencia los planteamientos de la declaración PRISMA (Urrútia y Bonfill, 2010).

\section{Estrategia de búsqueda}

La búsqueda de artículos publicados entre 2004 y 2014 se realizó en agosto de 2014 en las bases de datos; Springer Link, Scopus y ScienceDirect. Los términos utilizados en la búsqueda fueron: structure, operation, fertility island, fertile island, y agricultural, para su interrelación se utilizaron operadores boleanos bajo la siguiente sintaxis: ("structure" OR "operation") AND ("fertility islands" OR "fertile islands") AND ("agricultural").

Para el caso de la base de datos de Springer Link, se realizaron dos búsquedas independientes, en un primer momento con el término "fertility island" y posteriormente con "fertile island", ya que la consulta en esta base de datos limita la combinación de términos con operadores boleanos en la sintaxis.

La búsqueda de literatura gris, se complementó mediante las bases de datos de Tesis Doctorales en Red, DART Europe y Open Access Theses and Dissertations, sin que se reporten resultados entre 2004 y 2014, por tanto, la presente revisión no incluye publicaciones científicas no indexados.

La selección de los artículos que incluían elementos conceptuales relacionados con la estructura y operación de las islas de fertilidad, partió de los registros depurados en las bases de datos, para su posterior revisión en función de los criterios de inclusión y exclusión previamente determinados.

\section{Criterios de selección de estudios}

Los artículos seleccionados tomaron en cuenta artículos originales publicados en los últimos 10 años, escritos en idioma inglés, que incluyeran información sobre: i) estructura, procesos, componentes y operación de las islas de fertilidad, ii) características climáticas del área de estudio, especies de arbustos y gramíneas objeto de la investigación, factores abióticos, bióticos y de control, y iii) patrones espaciales y temporales 
de la dinámica de nutrientes en suelos áridos y conectividad con el paisaje adyacente.

Los artículos excluidos de la revisión, tomaron en cuenta los siguientes criterios: i) investigaciones sobre formación de islas de fertilidad como consecuencia de incendios forestales, y ii) estudios sobre metodologías de medición. Estos criterios fueron definidos luego de la lectura de todos los resúmenes de los artículos depurados.

\section{Recolección y consolidación de información}

La gestión de la información de cada una de las publicaciones se realizó en una hoja de datos de
Excel incluyendo datos generales, de descripción de la zona de estudio y elementos conceptuales (Tabla 1).

\section{Resultados y Discusión}

Un total de 315 artículos fueron identificados y tamizados en las diferentes etapas de la revisión sistemática (Figura 1), de los que 18 son incluidos parte la síntesis cualitativa (Tabla 2).

Los estudios principalmente analizaron temas relacionados con los nutrientes y su distribución espacio-temporal (6 estudios, 33\%), el rol de los arbustos en la formación de islas de fertilidad (5 estudios, 27\%), el impacto en el paisaje y el uso

Tabla 1. Datos para la consolidación de información de publicaciones.

\begin{tabular}{lll}
\hline Datos generales & \multicolumn{1}{c}{ Descripción de la zona de estudio } & \multicolumn{1}{c}{ Elementos conceptuales } \\
\hline 1. Título & 7. Lugar del estudio & 13. Suelo y nutrientes \\
2. Base de datos & 8. Clima & 14. Estructura \\
3. Revista & 9. Temperatura $\left({ }^{\circ} \mathrm{C}\right)$ máxima, mínima, promedio & 15. Función isla de fertilidad \\
4. Autor & 10. Potencial de evaporación $(\mathrm{mm})$ & \\
5. Año & 11. Textura del suelo & \\
6. País & 12. Vegetación de estudio & \\
\hline
\end{tabular}

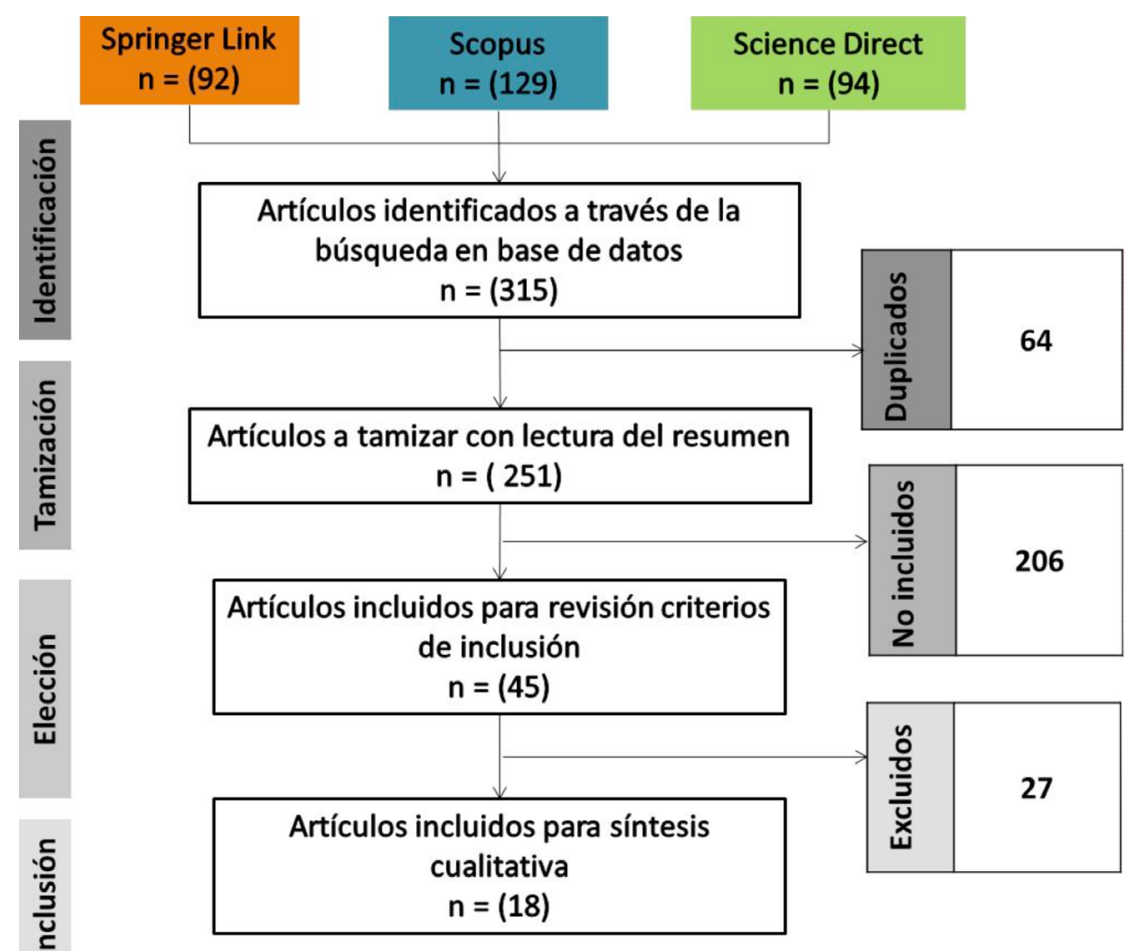

Figura 1. Etapas de la revisión sistemática. 
Alhamad y Alrababah

Tabla 2. Información general publicaciones incluidas en la revisión sistemática.

\begin{tabular}{rlll}
\hline $\mathrm{N}^{\text {o }}$ & \multicolumn{1}{c}{ Referencia } & \multicolumn{1}{c}{ Revista } & \multicolumn{1}{c}{ País } \\
\hline 1 & Allington y Valone (2013) & Ecosystems & Estados Unidos \\
2 & C. Li et al. (2013) & Environmental Earth Sciences & China \\
3 & Alhamad y Alrababah (2013) & Plant Ecology y Diversity & Jordan \\
4 & Xu et al. (2012) & Ecological Research & China \\
5 & Daryanto y Eldridge (2012) & Arid Environments & Australia \\
6 & Zaady et al. (2013b) & Arid Environments & Israel \\
7 & F.-R. Li et al. (2012) & Journal of Insect Conservation & China \\
8 & Zhang, Yang, Zhao, Bao, y Song (2011) & Plant and Soil & China \\
9 & Yang et al. (2011b) & Arid Environments & China \\
10 & DeMars et al. (2010) & Biological conservation & Estados Unidos \\
11 & Dossa et al. (2009) & Plant and Soil & Senegal \\
12 & Ruiz et al. (2008) & Arid Environments & México \\
13 & Caballero et al. (2007) & Plant Ecology & España \\
14 & Qinmei et al. (2007) & Acta Ecologica Sinica & China \\
15 & Orlando, Chávez, Bravo, Guevara, y Carú (2007) & Soil Biology y Biochemistry & Chile \\
16 & Housman et al. (2007) & Soil Biology y Biochemistry & Estados Unidos \\
17 & Manning et al. $(2006)$ & Biological conservation & Australia \\
18 & Huxman et al. (2004) & Oecología & Estados Unidos \\
\end{tabular}

del suelo (4 estudios, 22\%) y en menor medida los relacionados con el rol de mamíferos e insectos ( 2 estudios, $12 \%$ ) y los bancos de semilla asociados a las islas de fertilidad (1 estudio, 6\%).

La síntesis cualitativa incluye las investigaciones de nueve países (Figura 2), con una mayor representatividad de China con seis estudios y de Estados Unidos con cuatro.
Los datos de precipitación reportados en los estudios, se encuentran en el rango de 110-450 mm/ año, con temperaturas máximas que oscilan entre los 8 y $43,1^{\circ} \mathrm{C}$ y temperaturas mínimas entre -45 a $20^{\circ} \mathrm{C}$. Un total de 28 especies vegetales entre árboles, arbustos y gramíneas fueron reportadas en 15 investigaciones que incluyeron trabajo de campo.

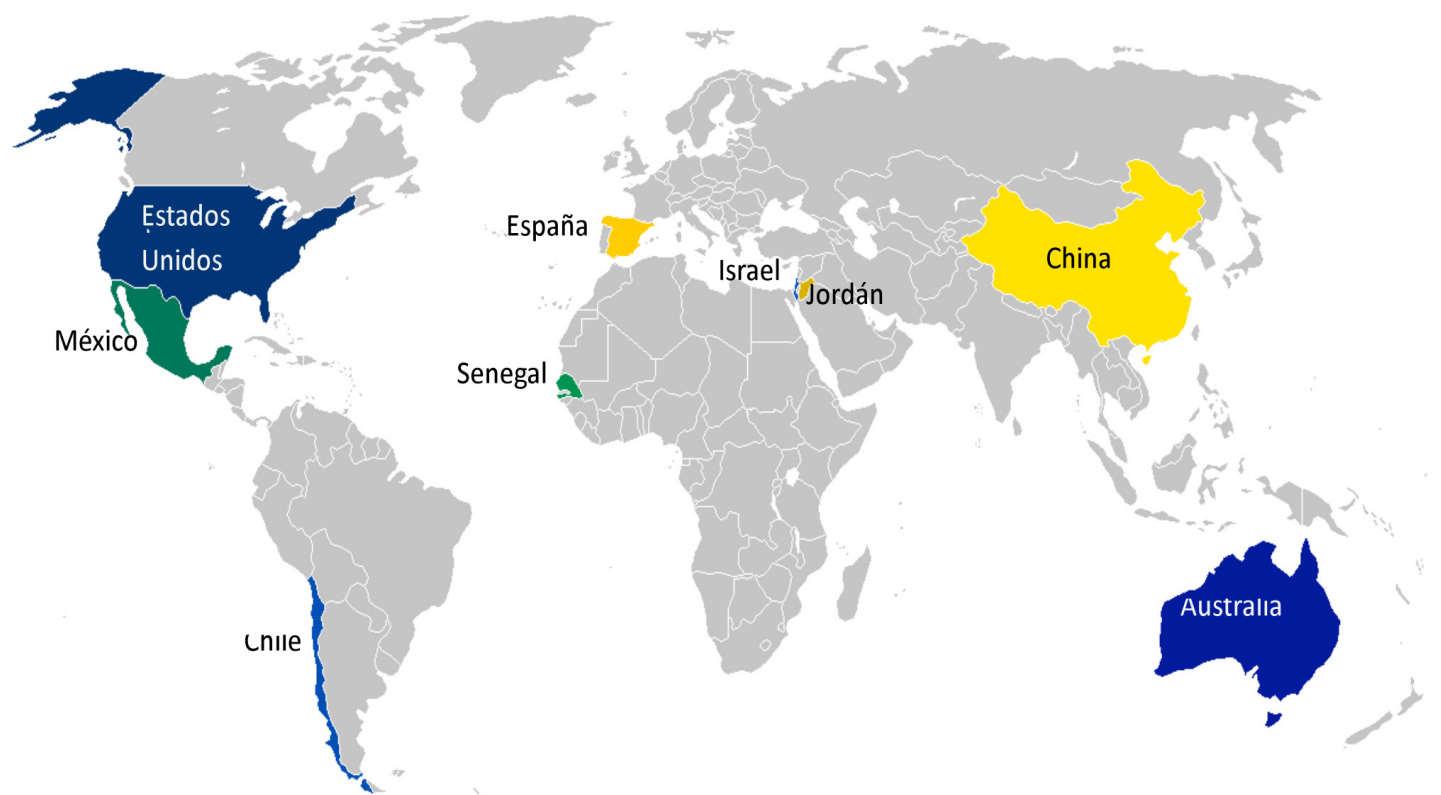

Figura 2. Mapa distribución geográfica estudios incluidos en la revisión sistemática. 


\section{Concepto de isla de fertilidad}

En zonas áridas o semiáridas las islas de fertilidad, o islas fértiles, o de recursos (Allington y Valone, 2013; Housman et al., 2007), son también consideradas islas de intensidad biogeoquímica (Dossa et al., 2009), y montículos fitogenéticos (Zhang et al., 2011), las que se forman debido al aporte potencial de nitrógeno de los arbustos cuando mueren, a la hojarasca y materia orgánica acumulada bajo el arbusto, y al nitrógeno disponible en la capa superficial del suelo (Garcia-Moya y McKell, 1970).

Esta definición concuerda con la propuesta por Stock et al. (1999), ya que refieren las islas de fertilidad como zonas de enriquecimiento de nutrientes bajo los arbustos, resultado de una serie de mecanismos de interacción físico, químico y biológico de concentración (Figura 3).

Sin embargo, Allington y Valone (2013), plantean discrepancias con las anteriores definiciones, proponiendo que las islas de fertilidad son una consecuencia del sobrepastoreo, de las prácticas agrícolas y del cambio climático. De acuerdo con Zaady et al. (2013), estas prácticas tienen un efecto residual a largo plazo ya que pueden afectar la superficie del suelo, la estructura y función del paisaje.

Adicionalmente, los autores sugieren que los parches de arbustos actúan como sumideros de la materia erosionada y la escorrentía generada en la superficie, siendo esta acumulación, lo que permite la transición de pastizales áridos o recientemente desertificados hacia vegetación de pastizales y, posteriormente a matorrales.

Estas dos concepciones de islas de fertilidad presentan discrepancias con en el origen y formación de las islas. Para Allington y Valone (2013), las islas de fertilidad son el resultado de procesos socioecológicos que evidencian la relación entre la formación de las islas con la historia del territorio y los procesos de toma de decisión. En contraposición a la visión de la isla de fertilidad como resultado de procesos físicos y biológicos en el que las plantas frecuentemente inducen acumulación de nutrientes bajo los parches de vegetación (Ruiz et al., 2008).

\section{Estructura de la isla de fertilidad}

Las islas de fertilidad están compuestas por parches y micro parches de vegetación rodeados

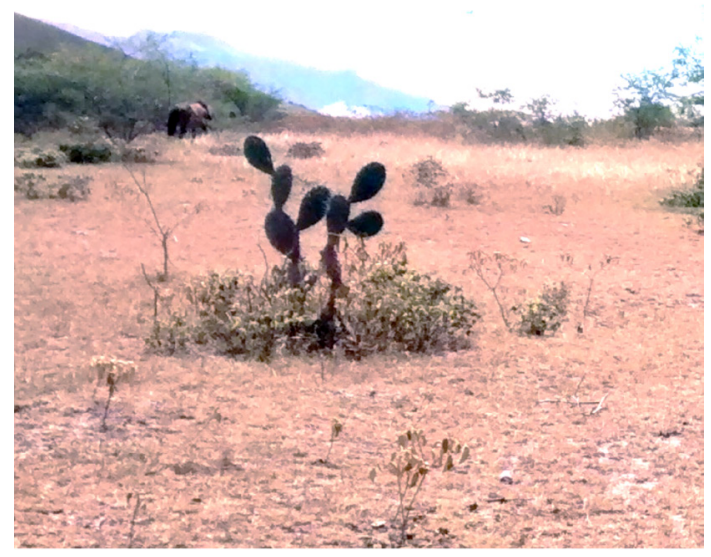

a) Sistema de vegetación simple inicio formación de la isla de fertilidad

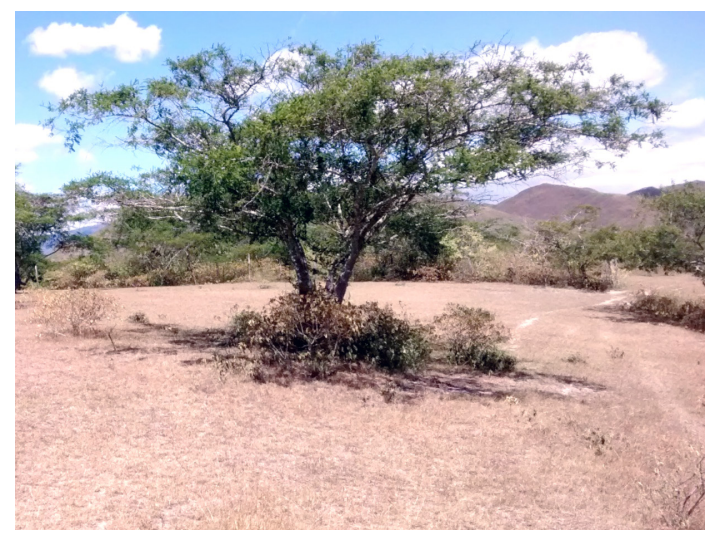

b) Zona de enriquecimiento de nutrientes bajo el arbusto

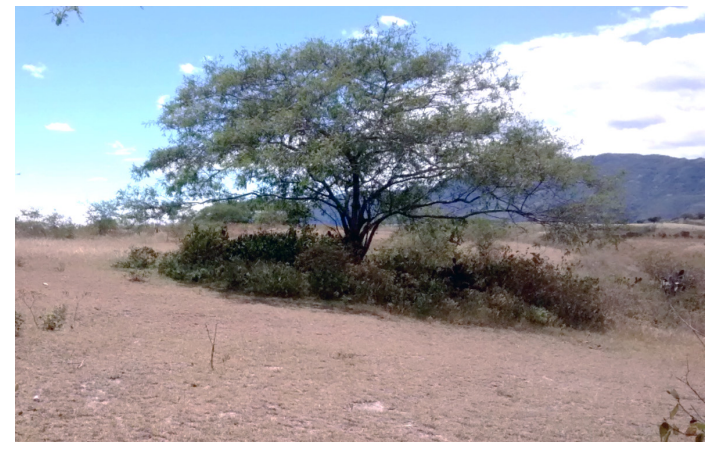

c) Isla de fertilidad

Figura 3. Islas de fertilidad zona semiárida Municipio de El Tambo Nariño, Colombia.

por una matriz de suelo descubierto, como un mosaico en dos fases que está compuesto por una capa discontinua de arbustos y un estrato herbáceo continuo (Caballero et al., 2007; Qinmei et al., 2007), las que integran microorganismos, comunidades de artrópodos y diferentes especies de animales. 
La capa de árboles y arbustos dispersos, de acuerdo con Alhamad y Alrababah (2013); Daryanto y Eldridge (2012), crea variabilidad en las características y condiciones de crecimiento de las plantas, según la distancia y aspecto de la copa o dosel. Estos hallazgos concuerdan con los estudios de Schlesinger y Pilmanis (1998), quienes sugieren que la vegetación dispersa influencia, la distribución de los nutrientes, la expresión superficial de otras propiedades del suelo y la creación de microparches. Además, de funcionar como "plantas nodrizas", que proporcionan condiciones favorables para el reclutamiento de otras plantas (Manning et al., 2006).

En cuanto a los microorganismos, Orlando et al. (2007) y Ruiz et al. (2008), coinciden en afirmar que su presencia facilita la asimilación de nutrientes, fijan nitrógeno, suprimen patógenos y permiten la dispersión de minerales. Por su parte, las comunidades de artrópodos, descomponedores y herbívoros ubicadas bajo los arbustos, contribuyen a mejorar las condiciones del suelo y el flujo de nutrientes al utilizar las fuentes de alimento generados por la hojarasca (F.-R. Li et al., 2012).

Otros estudios se enfocan en la presencia de pequeños mamíferos al interior de las islas, reportando que los animales al excavar madrigueras para protegerse y acaparar alimentos, promueven la concentración de nutrientes en el suelo, los que posteriormente tienen un efecto sobre la actividad microbiana, la calidad del suelo y la aceleración de la descomposición de materiales orgánicos, mejorando de esta manera, la infiltración del agua, la aireación y la distribución de materia orgánica en el perfil del suelo (Xu et al., 2012).

Las interacciones entre los estratos herbáceo, arbustivo, microorganismos, artrópodos, y fauna permiten, según Yang et al. (2011), que el sistema de vegetación simple, se transforme en un sistema de mayor complejidad ecológica.

\section{Funciones de la isla de fertilidad}

Las islas de fertilidad cumplen funciones estructurales y de soporte en ecosistemas áridos o semiáridos, que influyen sobre las propiedades del suelo, la protección ecológica y la conservación de la biodiversidad en diferentes escalas.

Con respecto a las funciones estructurales, las islas actúan como barrera física para la acumulación y mantenimiento de nutrientes y de la biomasa microbiana ((Li et al., 2012; Qinmei et al., 2007). Según los autores estos depósitos propician un ambiente más favorable a la vida, en términos de temperatura y humedad que se observa al interior de los parches de vegetación en ecosistemas áridos y semiáridos.

Adicionalmente, al comportarse como islas de intensidad biogeoquímica, permiten el aprovisionamiento de alimentos para herbívoros y mejoran la calidad forrajera de los pastos que se generan bajo los doseles debido a cambios en sus características morfológicas (Qinmei et al., 2007).

En cuanto a la protección ecológica, los parches de vegetación proporcionan una importante continuidad ecológica a través del tiempo ((Li et al., 2013; Zhang et al., 2011; Qinmei et al., 2007), ya que colaboran con el control de la erosión hídrica por la escorrentía, de la erosión eólica por el control de la velocidad de los vientos, y en la prevención de la pérdida de nutrientes y de la desertificación ((Li et al., 2013; Zhang et al., 2011; Qinmei et al., 2007).

Además de lo anterior, las islas de fertilidad según DeMars et al. (2010), cumplen la función de soporte de especies que contribuye a la conservación de la biodiversidad, ya que se desempeñan como estructuras claves del hábitat que proporcionan recursos críticos necesarios para la persistencia de especies. Por ejemplo, proveen a las aves de un espacio para percharse, forrajear o anidar. En el caso de mamíferos pequeños como jerbos (Rhombomys opimus) y conejos (Oryctolagus cuniculus) promueven la fertilidad del sistema y cumplen una función ecológica (Xu et al., 2012).

A una escala mayor, las islas de fertilidad según Yang et al. (2011) y Manning et al. (2006), también pueden operar como un punto central de la sucesión natural, debido a que tienen un rol dinamizador para la reconfiguración espacio-temporal a nivel de árbol, parche, corredor y paisaje.

Dicha sucesión guarda estrecha relación con la composición y densidad de los bancos de semillas, que propician el establecimiento de vegetación y que al mismo tiempo puede colaborar con la producción agrícola en estos ecosistemas (Caballero et al., 2007). Así, los árboles remanentes en los agroecosistemas son una estrategia de conservación regional debido a la función de soporte de especies que ofrecen en los paisajes agrícolas (DeMars et al., 2010). Por lo tanto, las islas de fertilidad configuran importantes reservas de nutrientes a 
nivel local y regional que pueden influenciar la estructura y funciones del ecosistema (C. Li et al., 2013), conformando un biopaisaje especial, como resultado de procesos físicos y biológicos (Huxman et al., 2004; Xu et al., 2012)..

Adicionalmente, las islas de fertilidad en zonas áridas y semiáridas pueden formarse por procesos ecológicos o socio-ecológicos, lo que implica una necesidad de establecer tipologías que permitan identificar su estructura y funcionamiento integrando los conocimientos generados desde las distintas ciencias. Asimismo, pueden ser estudiadas como indicadores de perturbación o restauración según el contexto del ecosistema. Este análisis permite tomar decisiones de conservación y gestión en los territorios orientadas a generar una matriz ecológica en ambientes con recursos limitados.

Desde la perspectiva de los agroecosistemas, las islas de fertilidad son importantes porque contribuyen con dos características fundamentales del suelo, el diseño y la calidad, ya que fomentan el incremento de la productividad y la restauración natural, que incide en el control biológico de plagas y enfermedades, mejorando la disponibilidad de nutrientes para las plantas y la relación de interconectividad entre las islas.
Desde la perspectiva de gestión del territorio, el diseño de agroecosistemas a partir de islas de fertilidad cumple un papel estratégico en la fertilidad del sistema, debido a la integración entre suelo, plantas y animales, tanto domésticos como no domesticados, que puedan fomentar el aporte de biomasa al sistema y la interconectividad de estos parches.

Finalmente, las islas de fertilidad y su relación con la agricultura, como estrategia para la reconfiguración de la matriz natural y productiva de los agroecosistemas de zonas áridas, ha sido poco estudiada y podría ser un tema de investigación que observa las relaciones socioecológicas en las escalas temporales y espaciales del territorio desde la unidad espacial de la isla hasta la de paisaje.

\section{Agradecimientos}

Los autores del manuscrito agradecen al $\mathrm{Sr}$. Vagner Lopes Da Silva por la colaboración en sus valiosos comentarios sobre versiones anteriores de este documento. También agradecemos al editor y a dos revisores anónimos por sus útiles comentarios.

\section{Literatura Citada}

Alhamad, M.N.; Alrababah, M.A.

2013. The impacts of biologically induced micro-environments on biodiversity in a dry Mediterranean grassland. Plant Ecology y Diversity, 6 (2): 279-288.

Allington, G.R.H.; Valone, T.J.

2013. Islands of Fertility: A Byproduct of Grazing? Ecosystems, 17 (1): 127-141.

Caballero, I.; Olano, J.M.; Escudero, A.; Loidi, J.

2007. Seed bank spatial structure in semi-arid environments: beyond the patch-bare area dichotomy. Plant Ecology, 195 (2): 215-223.

Daryanto, S. y Eldridge, D.J.

2012. Shrub hummocks as foci for small animal disturbances in an encroached shrubland. Journal of Arid Environments, 80: 35-39.

DeMars, C.A.; Rosenberg, D.K. y Fontaine, J.B.

2010. Multi-scale factors affecting bird use of isolated remnant oak trees in agro-ecosystems. Biological Conservation, 143 (6): 1485-1492.

Dossa, E.L.; Diedhiou, S.; Compton, J.E.; Assigbetse, K.B. y Dick, R.P.

2009. Spatial patterns of $P$ fractions and chemical properties in soils of two native shrub communities in Senegal. Plant and Soil, 327 (1-2): 185-198.

García-Moya, E. y McKell, C.E.

1970. Contribution of Shrubs to the Nitrogen Economy of a Desert-Wash Plant Community. Ecology, 51 (1): 81-88.
Housman, D.C.; Yeager, C.M.; Darby, B.J.; Sanford, R.L.; Kuske, C.R.; Neher, D.A. y Belnap, J.

2007. Heterogeneity of soil nutrients and subsurface biota in a dryland ecosystem. Soil Biology and Biochemistry, 39 (8): 2138-2149.

Huxman, T.E.; Snyder, K.A.; Tissue, D.; Leffler, A.J.; Ogle, K.; Pockman, W.T.; Potts, D.L.

2004. Precipitation pulses and carbon fluxes in semiarid and arid ecosystems. Oecologia, 141: 254-268.

Li, C.; Shi, X.; Lei, J. y Xu, X.

2013. The scale effect on the soil spatial heterogeneity of Haloxylon ammodendron (C.A. Mey.) in a sandy desert. Environmental Earth Sciences, 71 (9): 4199-4207.

Li, F.-R.; Liu, J.-L.; Liu, C.-A.; Liu, Q.-J. y Niu, R.-X.

2012. Shrubs and species identity effects on the distribution and diversity of ground-dwelling arthropods in a Gobi desert. Journal of Insect Conservation, 17 (2), 319-331.

Manning, A.D.; Fischer, J. y Lindenmayer, D.B.

2006. Scattered trees are keystone structures - Implications for conservation. Biological Conservation, 132 (3), 311-321.

Orlando, J.; Chávez, M.; Bravo, L.; Guevara, R.; Carú, M.

2007. Effect of Colletia hystrix (Clos), a pioneer actinorhizal plant from the Chilean matorral, on the genetic and potential metabolic diversity of the soil bacterial community. Soil Biology and Biochemistry, 39 (11): 2769-2776.

Qinmei, Z.; Cheng, Z.; Maosong, L.; Wen, Y.; Chi, X.; Hanjie, W. 2007. The influences of arboraceous layer on spatial patterns and morphological characteristics of herbaceous 
layer in an arid plant community. Acta Ecologica Sinica, 27 (4): 1265-1271.

Ruiz, T.G.; Zaragoza, S.R.; Cerrato, R.F.

2008. Fertility islands around Prosopis laevigata and Pachycereus hollianus in the drylands of Zapotitlán Salinas, México. Journal of Arid Environments, 72 (7): 1202-1212. Schlesinger, W. H.; Pilmanis, A.M.

1998. Plant-soil interactions in deserts. Biogeochemistry, 42 (1): 169-187.

Stock, W.D.; Dlamini, T.S.; Cowling, R.M.

1999. Plant Induced Fertile Islands as Possible Indicators of Desertification in a Succulent Desert Ecosystem in Northern Namaqualand, South Africa. Plant Ecology, 142 (1): 161-167.

Urrútia, G.; Bonfill, X.

2010. PRISMA declaration: a proposal to improve the publication of systematic reviews and meta-analyses. Medicina Clínica, 135 (11): 507-11.
Xu, W.; Liu, W.; Yang, W.; Tang, C.; Blank, D.

2012. Rhombomys opimus contribution to the "fertile island" effect of tamarisk mounds in Junggar Basin. Ecological Research, 27 (4): 775-781.

Yang, Z.P.; Zhang, Q.; Wang, Y.L.; Zhang, J.J.; Chen, M.C.

2011. Spatial and temporal variability of soil properties under Caragana microphylla shrubs in the northwestern Shanxi Loess Plateau, China. Journal of Arid Environments, 75 (6): 538-544.

Zaady, E.; Arbel, S.; Barkai, D.; Sarig, S.

2013. Long-term impact of agricultural practices on biological soil crusts and their hydrological processes in a semiarid landscape. Journal of Arid Environments, 90: 5-11.

Zhang, P.; Yang, J.; Zhao, L.; Bao, S.; Song, B.

2011. Effect of Caragana tibetica nebkhas on sand entrapment and fertile islands in steppe-desert ecotones on the Inner Mongolia Plateau, China. Plant and Soil, 347 (1-2): 79-90. 